\title{
Evaluation of Chemotherapy Drug Exposure in a Veterinary Specialty Hospital
}

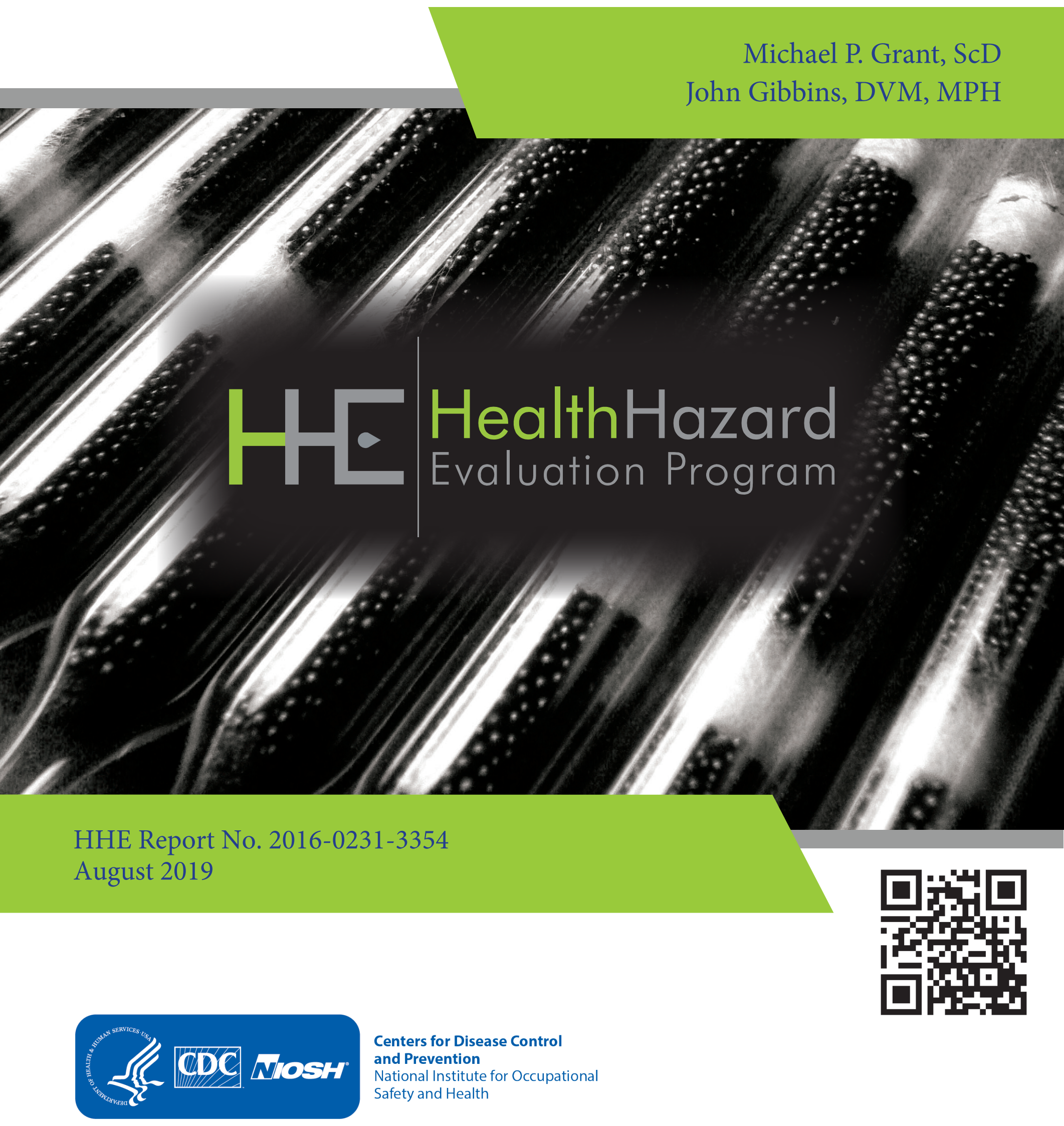




\section{Contents}

Highlights.

Abbreviations ...................................... iii

Introduction ............................................ 1

Methods .............................................. 1

Results and Discussion ........................ 2

Conclusions ....................................... 14

Recommendations............................. 15

Appendix A .......................................... 18

Appendix B ...................................... 19

References........................................ 21

Acknowledgements............................ 25

The employer is required to post a copy of this report for 30 days at or near the workplace(s) of affected employees. The employer must take steps to ensure that the posted report is not altered, defaced, or covered by other material.

The cover photo is a close-up image of sorbent tubes, which are used by the HHE Program to measure airborne exposures. This photo is an artistic representation that may not be related to this Health Hazard Evaluation. Photo by NIOSH. 


\section{Highlights of this Evaluation}

The Health Hazard Evaluation Program received a confidential request from a veterinary specialty hospital. The requestors were concerned about possible exposures to chemotherapy drugs and zoonotic diseases (infections shared between patients and people) for employees working in the chemotherapy administration area.

\section{What We Did}

- We visited the veterinary hospital in November 2016.

- We observed areas where chemotherapy drugs were received, stored, administered, and disposed.

- We observed patient intake, workup, chemotherapy administration, and discharge.

- We reviewed employer training materials and work-related illness and injury logs and reports.

- We held confidential interviews with employees who discussed work and medical histories, health and safety training and awareness, and perceptions about the work environment.

- We collected surface wipe samples for several chemotherapy drugs.

\section{What We Found}

- We found no chemotherapy drugs on any tested surfaces in the chemotherapy administration area.

- We found a small amount of cyclophosphamide on the floor just outside of the door to the

We evaluated employees' exposures to chemotherapy drugs and zoonotic diseases in a veterinary specialty hospital. We took surface samples for commonly administered chemotherapy drugs. We did not find contamination on any surfaces in the chemotherapy administration area. Employees did not report health concerns. We recommended refresher training and purchasing face shields to wear when handling hazardous drugs. chemotherapy administration area.

- Employees did not report any health concerns or symptoms related to handling and administering chemotherapy to patients.

- Employees did not report prior known infection from a variety of zoonotic diseases, such as salmonellosis, leptospirosis, or parasitic infections.

- Most employees were very satisfied with the hospital's current health and safety program, and reported wearing required personal protective equipment when working with chemotherapy drugs.

- New employees went through a comprehensive chemotherapy training program.

- Patient bites and scratches were the most common entry on the Occupational Safety and Health Administration (OSHA) Form 300 Log of Work-Related Injuries and Illnesses. 


\section{What the Employer Can Do}

- Continue the recent change in laundry practices where new yellow towels are used strictly for the chemotherapy administration area and are not placed into general circulation after laundering.

- Place a chemotherapy waste container in the laundry room. The employee doing laundry will no longer need to walk through the facility to dispose of chemotherapy waste, reducing the chance of them contaminating the facility.

- Provide a cart for employees to move chemotherapy drugs from shipping and receiving to the chemotherapy administration area.

- Require all employees to wear gloves that are protective against chemotherapy drugs whenever handling chemotherapy drugs.

- Continue rigorously training newer employees and begin comparable refresher training for employees who began work prior to the establishment of this comprehensive chemotherapy training program.

- Provide face shields in place of respirators to facilitate splash protection during chemotherapy administration.

- Discontinue use of N95 respirators. Prepare chemotherapy drugs in the biologic safety cabinet and administer via closed system transfer device.

- Follow OSHA guidelines for recording reportable events on the OSHA Logs. Not all patient bites and scratches require reporting on this form.

\section{What Employees Can Do}

- Use a cart to move new shipments of chemotherapy drugs from shipping and receiving to the chemotherapy administration area.

- Report any workplace health and safety concerns to the employer.

- Wear all required personal protective equipment when handling chemotherapy drugs.

- Attend refresher trainings and ask for training if needed. 


\section{Abbreviations}

$\begin{array}{ll}\text { ACGIH }^{\circledR} & \text { American Conference of Governmental Industrial Hygienists } \\ \text { BSC } & \text { Biosafety cabinet } \\ \text { CFR } & \text { Code of Federal Regulations } \\ \mathrm{cm}^{2} & \text { Centimeters squared } \\ \text { CSTD } & \text { Closed system transfer device } \\ \text { MRSA } & \text { Methicillin-resistant staphylococcus aureus } \\ \text { NIOSH } & \text { National Institute for Occupational Safety and Health } \\ \text { OEL } & \text { Occupational exposure limit } \\ \text { OSHA } & \text { Occupational Safety and Health Administration } \\ \text { PEL } & \text { Permissible exposure limit } \\ \text { PPE } & \text { Personal protective equipment } \\ \text { REL } & \text { Recommended exposure limit } \\ \text { STEL } & \text { Short-term exposure limit } \\ \text { TLV } & \text { Threshold limit value } \\ \text { TWA } & \text { Time-weighted average }\end{array}$


This page left intentionally blank 


\section{Introduction}

The Health Hazard Evaluation Program received a confidential request from a veterinary specialty hospital. The requestors were concerned about possible exposures to chemotherapy drugs and zoonotic diseases for employees working in the chemotherapy administration area. We visited the veterinary hospital in November 2016 and shared our preliminary findings, observations, and recommendations with the employer and employee representatives. We observed outpatient chemotherapy treatment for ten patients during our two-day visit. The specialty hospital employed 3 veterinary oncologists and 14 technical staff who worked with chemotherapy drugs. We summarized our site visit and made initial recommendations in a letter to the employer and employee representatives in December 2016. We also provided resources on good infection control practices to the hospital training coordinator by email in December 2016.

\section{Methods}

Our main objectives were to assess whether (1) employees understood and followed established protocols for the safe administration of hazardous drugs (from drug receipt through waste disposal); (2) work surfaces were contaminated with chemotherapy drugs; (3) employees were concerned about exposure to chemotherapy drugs and zoonotic diseases; and (4) employees had health symptoms or medical conditions associated with exposures to hazardous drugs or reported prior infections with zoonotic diseases.

\section{Surface Wipe Samples}

We collected a limited number of surface wipe samples for carboplatin (analyzing for total platinum), vinblastine, doxorubicin, cyclophosphamide, and vincristine (Table 1). We selected these drugs based on (1) the frequency that they were purchased in the current calendar year, (2) their presence on the National Institute for Occupational Safety and Health (NIOSH) hazardous drug list [NIOSH 2016], and (3) the availability of a sampling and analytical method. We selected wipe sample locations based on findings from previous health hazard evaluations, the work practices of employees, and other research [Connor and McDiarmid 2006; Connor et al. 2010; NIOSH 2012, 2014].

We sampled surfaces in the chemotherapy administration area after drug administration. We sampled the inside of a laundry bag, near the opening, after the laundry was loaded and before bag disposal. We sampled the outside of the chemotherapy transfer bag that a new shipment of chemotherapy drugs had arrived in after the bag was brought from shipping and receiving into the chemotherapy administration area. For each sample, we wore a clean pair of chemotherapy protective gloves and used a new 100 square centimeter $\left(\mathrm{cm}^{2}\right)$ disposable template to determine the sampling area. We used Alpha ${ }^{\circledR}$ texwipe swabs moistened with a $50 \%$ methanol, $50 \%$ water solution for the samples. We wiped the template area once horizontally with one side of the texwipe and then vertically with the other side. 
Table 1. Sampling and analytical methods for chemotherapy drugs in surface samples

\begin{tabular}{lccc}
\hline Chemotherapy drug & $\begin{array}{c}\text { Surface wipe/ } \\
\text { wetting agent }\end{array}$ & $\begin{array}{c}\text { Surface } \\
\text { area } \\
\text { sampled }\end{array}$ & $\begin{array}{c}\text { Analysis method } \\
\text { (Bureau Veritas North } \\
\text { America Internal Method) }\end{array}$ \\
\hline $\begin{array}{l}\text { Carboplatin } \\
\text { (reported as total } \\
\text { platinum) }\end{array}$ & $\begin{array}{c}\text { One Alpha texwipe swab } \\
\text { moistened with a } \\
50 \% \text { methanol, } 50 \% \text { water } \\
\text { solution }\end{array}$ & $100 \mathrm{~cm}^{2}$ & $\begin{array}{c}\text { Inductively coupled } \\
\text { plasma/mass spectrometry }\end{array}$ \\
$\begin{array}{l}\text { Doxorubicin } \\
\text { Cyclophosphamide }\end{array}$ & $\begin{array}{c}\text { One Alpha texwipe swab } \\
\text { moistened with a }\end{array}$ & $100 \mathrm{~cm}^{2}$ & $\begin{array}{c}\text { High performance liquid } \\
\text { chromatography }\end{array}$ \\
$\begin{array}{l}\text { Vincristine } \\
\text { Vinblastine }\end{array}$ & $\begin{array}{c}50 \% \text { methanol, } 50 \% \text { water } \\
\text { solution }\end{array}$ & & \\
\hline
\end{tabular}

\section{Employee Interviews and OSHA Injury/IIIness Logs}

We interviewed 15 of the 17 employees who gave chemotherapy drugs to patients. During these voluntary, confidential health interviews, we asked about work history, handling of chemotherapy drugs, medical histories, health and safety training and awareness, and perceptions about the work environment. We reviewed OSHA Logs for years 2013-2016.

\section{Review of Hazardous Drug Training and Procedures}

We reviewed training materials and checklists for licensed technicians and veterinary aides as well as continuing education materials for all staff who work with chemotherapy drugs. We observed patient intake, workup, chemotherapy administration, and discharge. We focused our observations on areas where chemotherapy drugs were received, stored, and administered. We reviewed the most recent report from the Class II biosafety cabinet (BSC) servicing from June 2016.

\section{Results and Discussion}

\section{Observations}

All chemotherapy drugs were generally ordered as needed and delivered to a central shipping and receiving area in the building. When packages came in, an employee donned nitrile gloves and opened the package. The employee checked to make sure that the product matched the packing slip and that the bottle had no damage. A highly visible spill kit was located on a shelf in the shipping and receiving area (Figure 1). This spill kit may be stored too high because contamination could run from the hands down the arms if an employee was contaminated and needed to reach up to access the kit. 
After checking the package, we observed the employee walking the package through the building to the chemotherapy administration area (Figure 2). The path to the chemotherapy administration area required opening two or three doors depending on whether one was propped open or not. The employee opened the doors with one hand and one elbow while holding onto the package with the other hand.

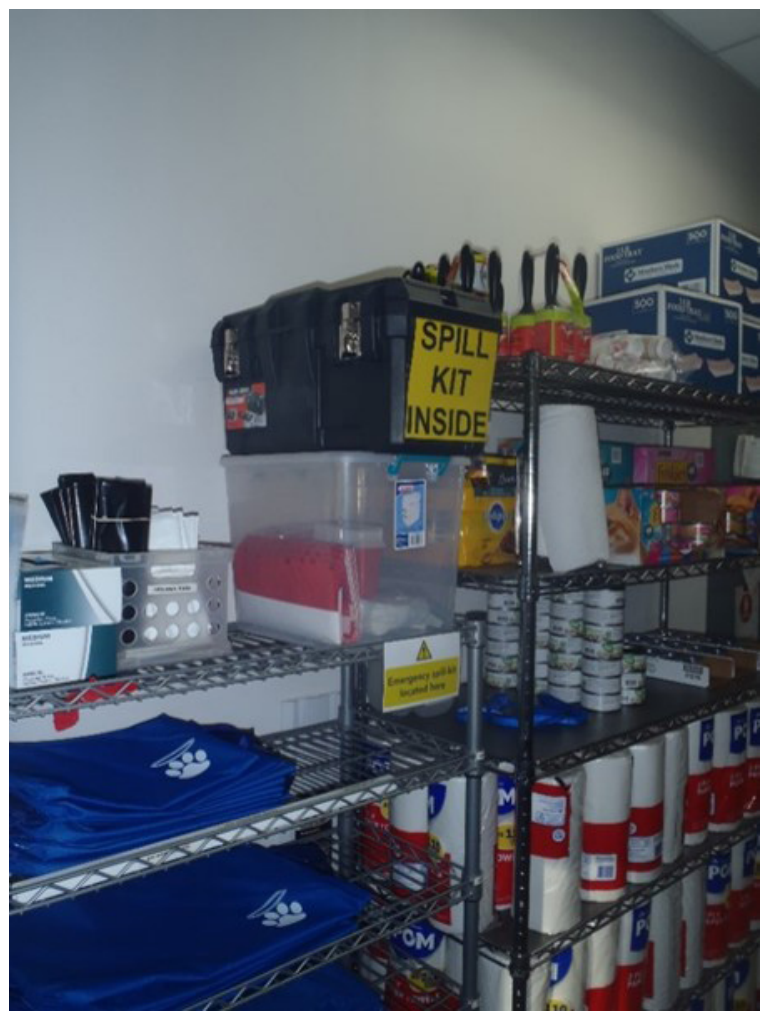

Figure 1. A spill kit in the shipping and receiving area. Photo by $\mathrm{NIOSH}$. 


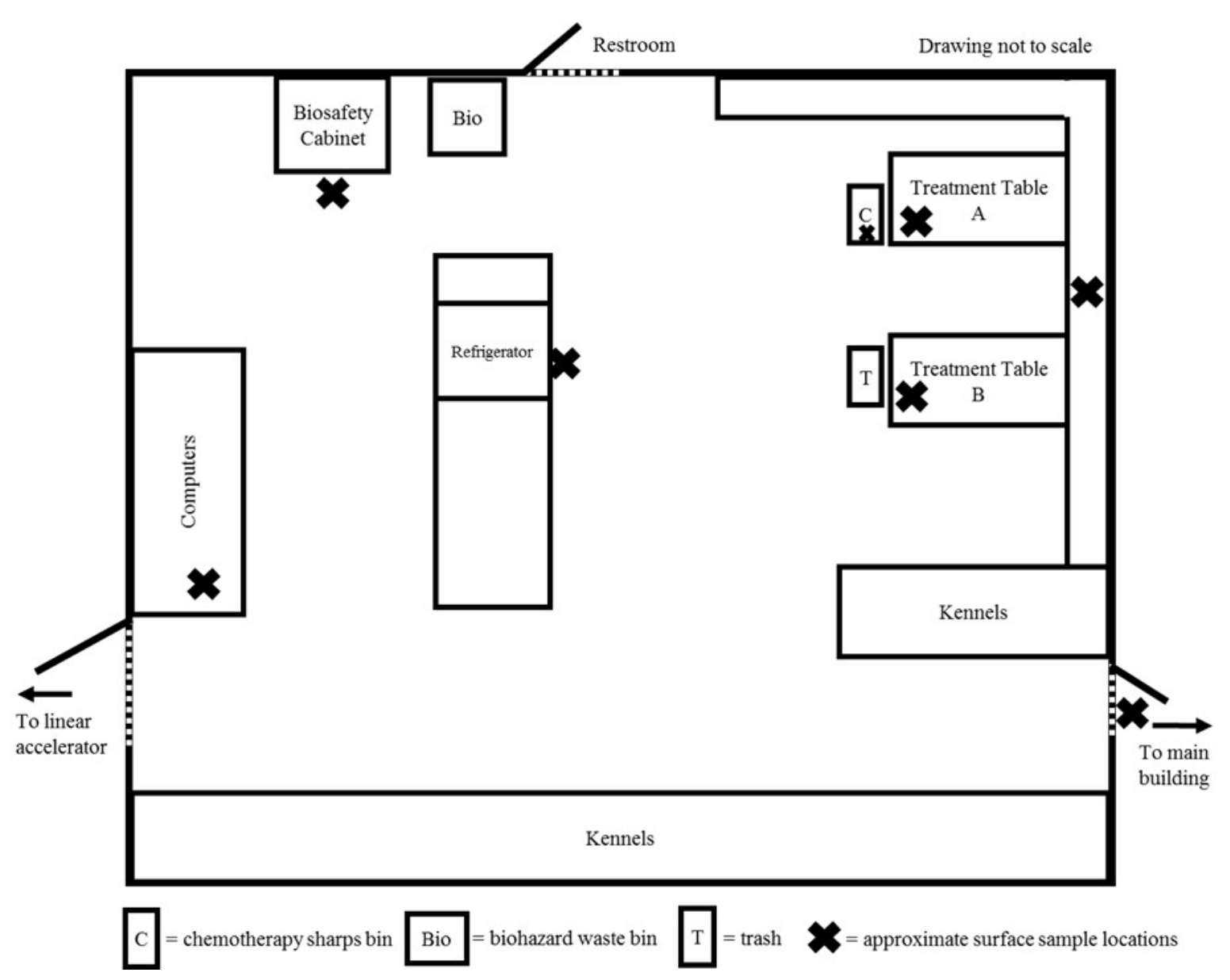

Figure 2. Diagram of the chemotherapy administration area.

Once in the chemotherapy administration area, the drugs were stored either in the refrigerator or cabinet according to label directions. The refrigerator was labeled with a large sign indicating that chemotherapy drugs were inside. A dedicated storage area is required for hazardous drugs [NIOSH 2004]. A dedicated refrigerator is required if hazardous drugs require refrigeration [USP 2019]. Opened drugs were fitted with a closed system transfer device (CSTD) as soon as they were opened. The CSTD was permanently attached to the bottle and never removed. The entire bottle and device were disposed of when empty. When we opened the refrigerator, we saw a stored bottle hanging from the wire rack in the refrigerator by the cap of the CSTD (Figure 3). 


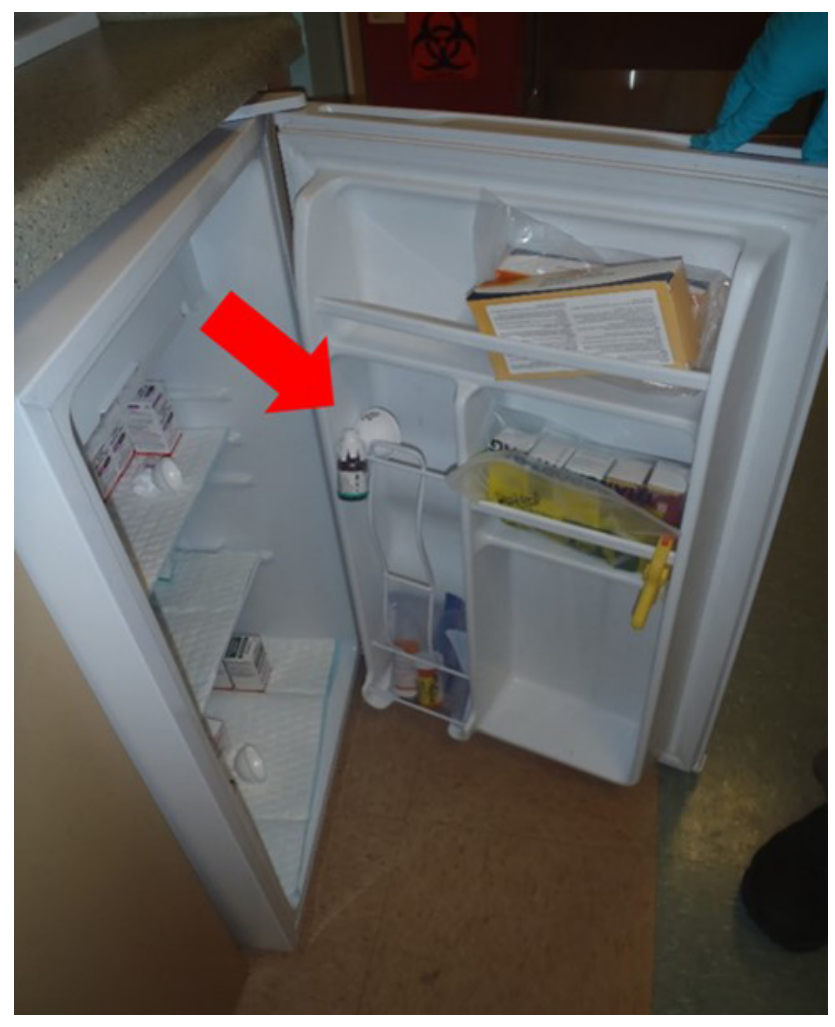

Figure 3. A bottle of chemotherapy drug hung from the refrigerator door by the cap of the closed system transfer device. Photo by NIOSH.

Employees wore disposable chemotherapy gowns, safety glasses, double nitrile gloves, and N95 respirators during drug preparation and administration. The nitrile gloves were not rated for use with chemotherapy drugs. Respirators were opened and used for a period of one month. Employees opened a respirator, wrote their initials and start date on the front in permanent marker, and hung them on a hook (Figure 4). While stored, the outside of one respirator touched the inside of the respirator hanging in front of it. The outside of a respirator can become contaminated during use. Touching the contaminated face of a respirator to the inside of another respirator could transfer contamination to the inside of the other respirator. 


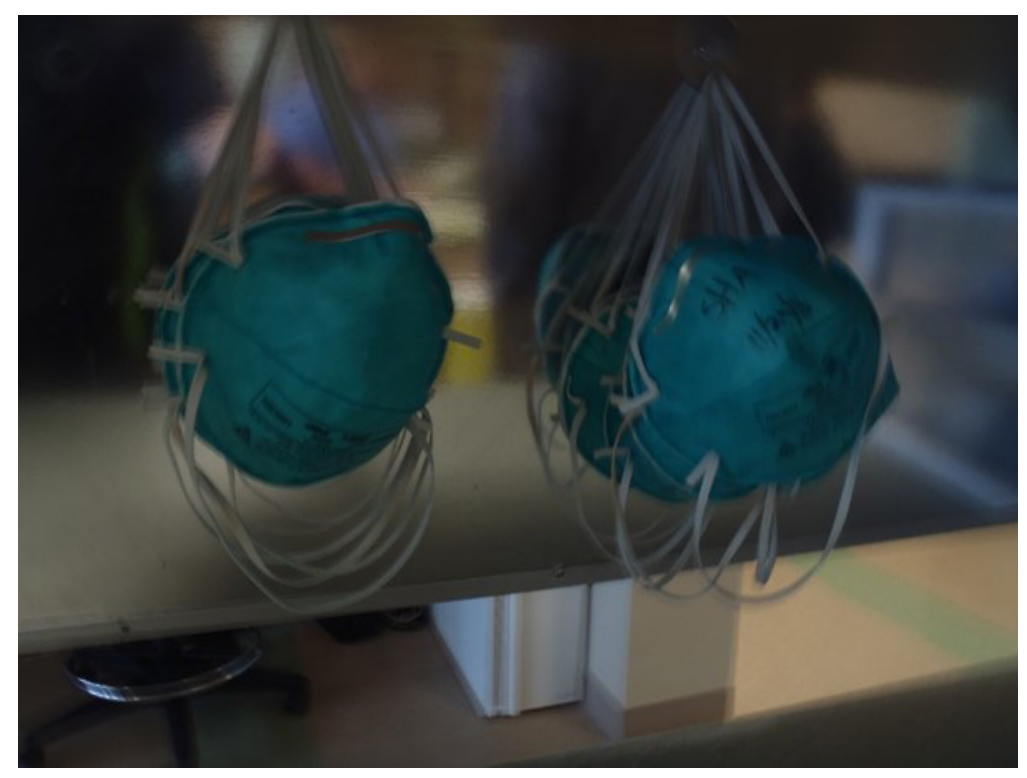

Figure 4. N95 respirators hung in the chemotherapy administration area. Photo by NIOSH.

In general, all chemotherapy drugs were prepared before the first appointment of the day. During our visit, the specialty hospital administered doxorubicin, mechlorethamine, carboplatin, lomustine, vinblastine, and vincristine. Mechlorethamine was administered to one patient during our visit although it was typically used only three or four times per year. For each patient, employees gathered the necessary components of the CSTD, the drug bottle, and a chemotherapy transport bag. Drug delivery syringes were prepared individually in a Class II BSC, which directly exhausted to the outside of the building. Employees turned on the BSC in the morning and then turned it off in the evening after all appointments had concluded and the chemotherapy administration area had been cleaned. We were told that only certain employees were responsible for turning the system on and off because the process involved two switches that had to be flipped in the correct order. The BSC was serviced semiannually and was recently certified to be operating according to manufacturers' specifications.

The components for the CSTD, the drug bottle, and a chemotherapy transport bag were placed in the BSC (Figure 5). Employees used the CSTD to draw the appropriate amount of drug into the syringe and then placed the prepared dose and the original bottle into the chemotherapy transport bag. The bag was then placed on a nearby treatment table. This was repeated until the drugs for all patients were prepared and placed on the treatment table (Figure 6). When the employee finished drug preparation, the bags were moved into the BSC until they were needed. During the preparation process, we observed a chemotherapy transport bag placed so that it covered part of the grill on the front of the BSC (Figure 5). Blocking any part of the grill interferes with the airflow and could allow contaminants into the room. 


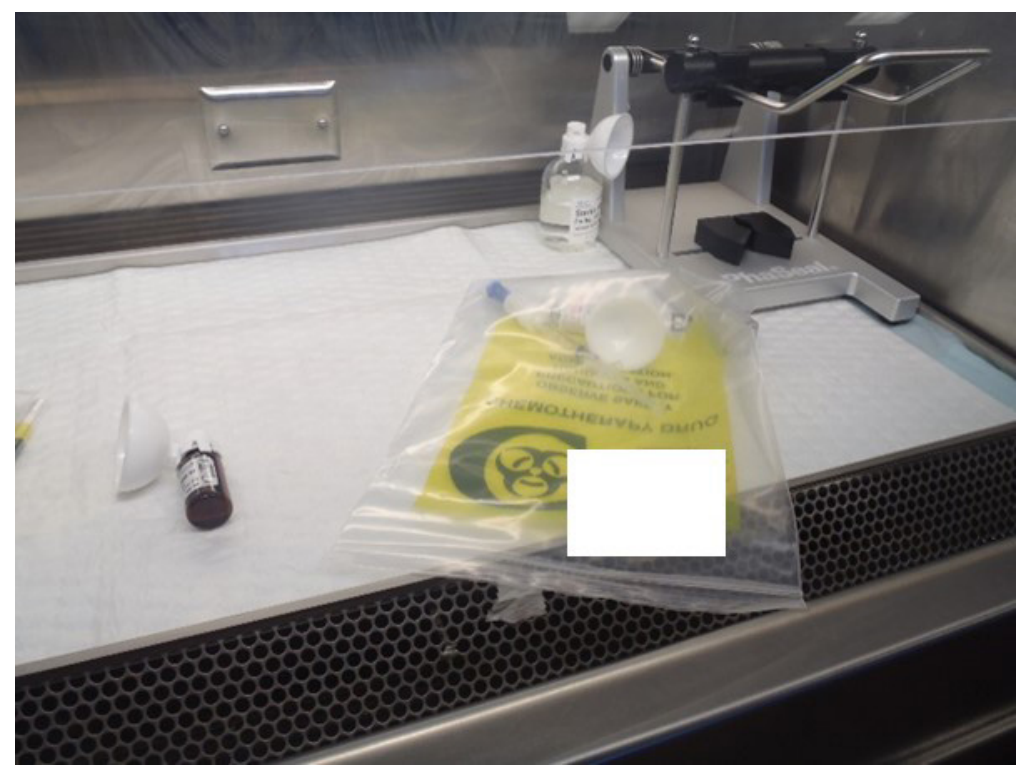

Figure 5. Chemotherapy drug preparation. Photo by NIOSH.

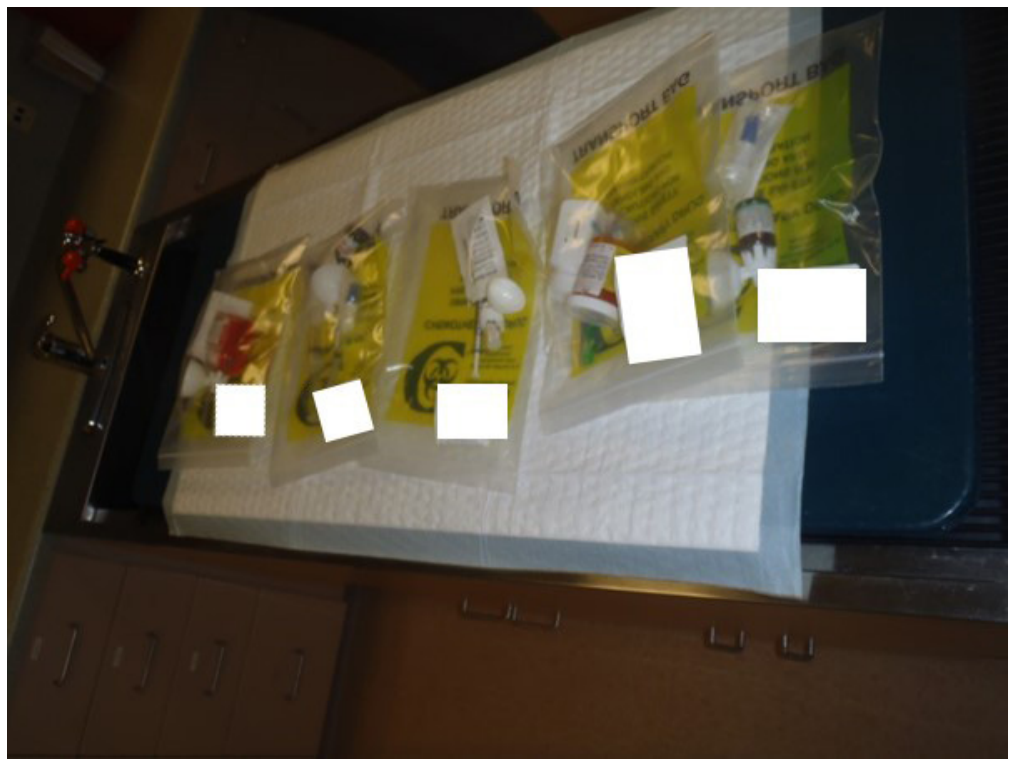

Figure 6. Chemotherapy drugs prepared and ready for administration. Photo by NIOSH. 
Patients were either placed on disposable, waterproof mats on treatment tables or on the floor depending on their size. A double check system was in place where a technician verified the patient, drug, and dose before the oncologist did the same. After confirmation, the chemotherapy was administered through the CSTD (Figure 7). At least two employees were involved with administration - one to hold the patient and one to administer the drug. After completing treatment, patients were kenneled for a period before discharge. They were usually discharged the same day as chemotherapy administration. All patients that have received chemotherapy within the prior 48 hours had a chemotherapy sticker on their cage card to notify employees of exposure risk. Patients were discharged to their owners with written instructions on how to safely handle the patient's urine, feces, and vomit that could be contaminated with chemotherapy drugs. The owners were also told how to safely clean up accidents and what precautions to take.

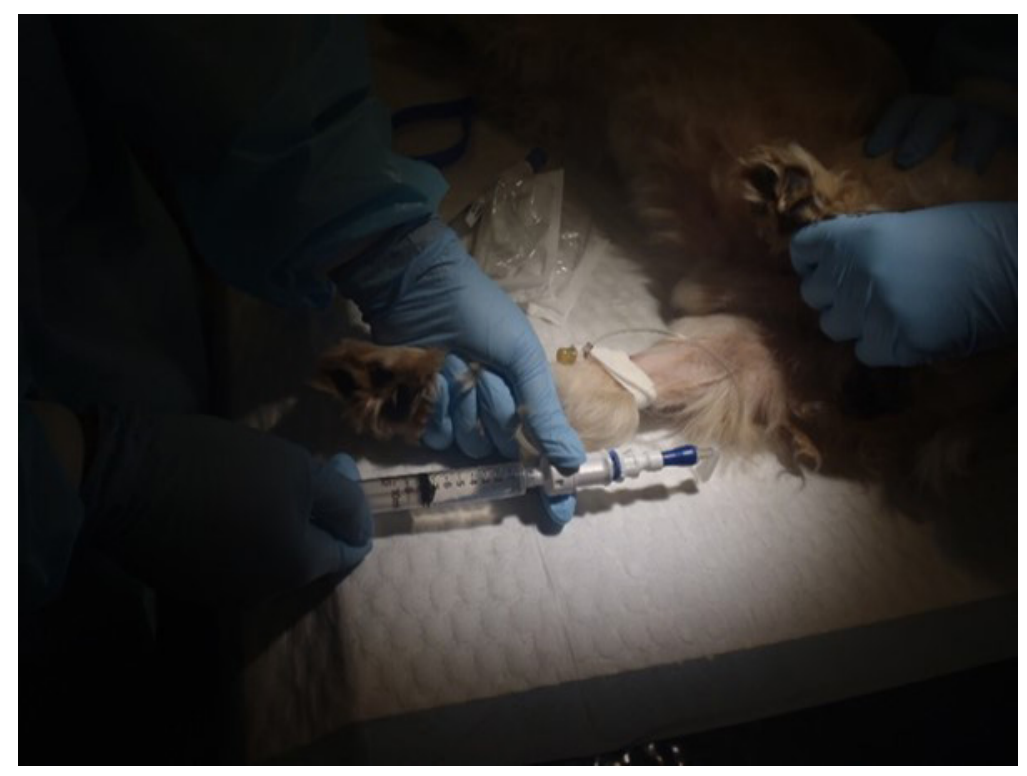

Figure 7. Chemotherapy drug administration. Photo by NIOSH.

Once the drug was administered, the bottle containing the drug was returned to storage. The syringe, CSTD components, tubing, and needle were placed back inside the chemotherapy transport bag and then disposed of in the chemotherapy sharps container. Employees removed their gloves and washed their hands with soap and water between patients. If a chemotherapy drug needed to be deactivated before disposal (e.g., mechlorethamine), the chemotherapy transport bag was placed in the BSC with the bottle while it was being deactivated (Figure 8). After deactivation, the bottle was placed in the transport bag and then disposed of in the chemotherapy sharps container. Between patients and at the end of the day, employees cleaned the treatment tables by spraying a 70\% isopropyl alcohol on surfaces and then wiping up with paper towels. 


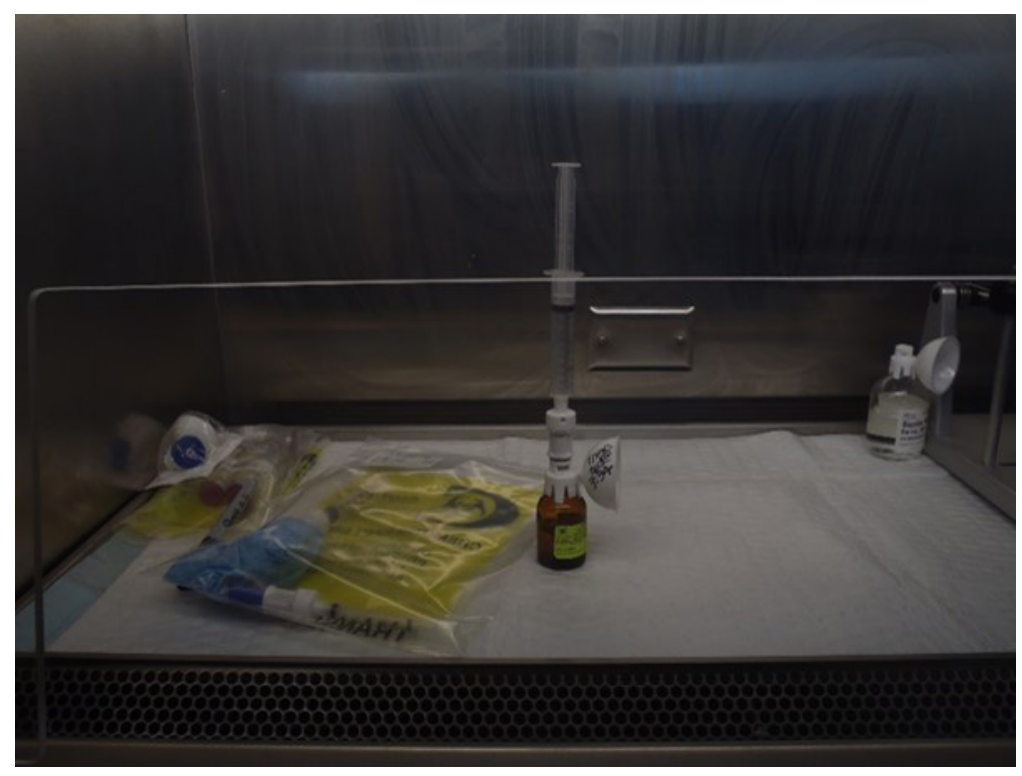

Figure 8. Deactivation of mechlorethamine in the biosafety cabinet. Photo by $\mathrm{NIOSH}$.

We observed hospital staff collect a fine needle aspirate of fluid for diagnostic purposes. The fluid was placed on a glass microscope slide for analysis. When finished, the employee disposed of the slide in the regular trash. The fluid on the slide may be contaminated with chemotherapy drugs because the patient had received chemotherapy in the past. Furthermore, the corners of the glass could tear the trash bag and presents an exposure and safety risk from potentially being cut by the glass slides.

We observed housekeepers cleaning floors, windows, and glass doors periodically throughout the day. The chemotherapy administration area was mopped by housekeeping at the end of the day after all patients had been discharged and the technicians had cleaned the treatment tables. We did not observe the housekeeping employee wearing any special personal protective equipment (PPE) when cleaning the chemotherapy administration area. We did not observe this employee taking out the trash. Housekeeping employees should be wearing disposable gowns and chemotherapy resistant gloves when working in the chemotherapy administration area [NIOSH 2004, 2016; USP 2019]. Employees should never push the air out of trash bags before removing them from the trash cans. Forcing air out of the bag could expose employees to airborne chemotherapy drugs. Employees should never spray cleaners directly onto potentially contaminated surfaces. Spray cleaning products onto paper towels and then wipe the potentially contaminated surface.

Chemotherapy laundry bags were moved to the laundry room when they were full (Figure 9). Prior to our visit, any towels used on chemotherapy patients were washed separately from general laundry and then returned into the general towel circulation throughout the hospital. It was possible for a patient in another part of the hospital to end up using a towel that had previously been used by a chemotherapy patient, laundered, and then put back into the clean towel supply. Just prior to our visit, the specialty hospital adopted a new chemotherapy laundry policy based on discussions with our team. The specialty hospital now uses yellow towels in the chemotherapy area; these are washed separately and only 
used in the chemotherapy area. This gives a visual clue to employees that the towel is from a chemotherapy patient. It also helps maintain separation of clean towels so that the clean chemotherapy laundry does not end up being used for the general population.

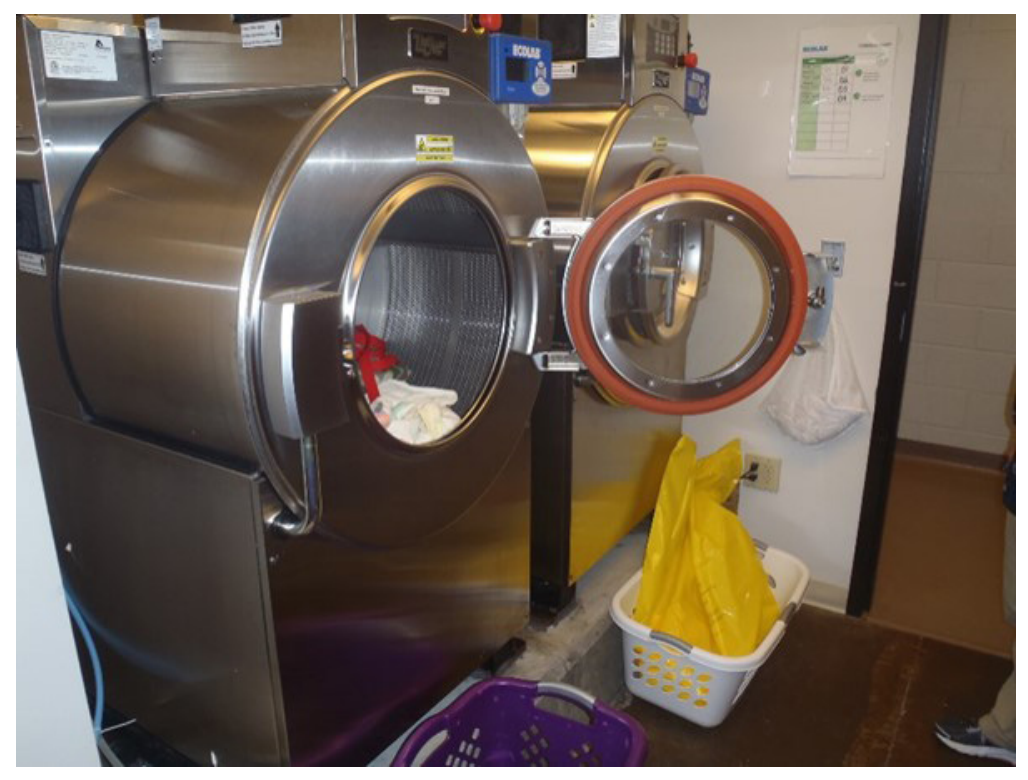

Figure 9. Chemotherapy laundry bag in the laundry room. Photo by $\mathrm{NIOSH}$.

During our visit, we observed the setup of the two isolation rooms for patients with suspected or confirmed zoonotic diseases. In addition to physical separation from the general population, employees placed a 1:10 bleach to water solution footbath at the doorway where employees were required to step in before leaving the room. When not in use, the footbaths were stored inside the isolation room along the wall (Figure 10). Limited data exist on the effectiveness of footbaths and foot mats in infection control although they are used in large patient veterinary teaching hospitals [Stull et al. 2018]. 


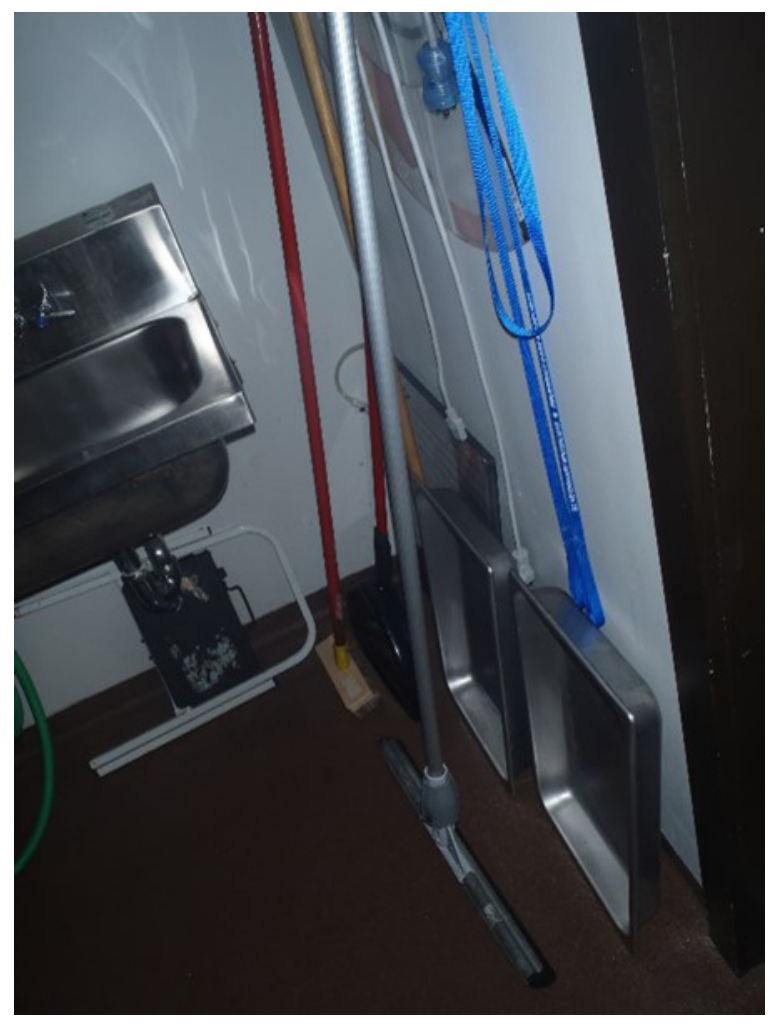

Figure 10. Footbath pans were stored along the wall inside the door to the isolation room where patients suspected to have communicable zoonotic diseases were examined. Photo by NIOSH.

\section{Surface Wipe Samples}

Detailed surface wipe sampling results can be found in Appendix A (Table A1). We did not detect any of the chemotherapy drugs we sampled for in the chemotherapy administration area or in the inside of a laundry bag. We also did not detect any drugs on the outside of a chemotherapy transport bag used to ship a new bottle of chemotherapy drug to the specialty hospital.

We detected cyclophosphamide on the floor just outside the chemotherapy administration area. This could suggest that cyclophosphamide was stable in the environment and that housekeeping procedures were not effectively removing it. In a previous evaluation at an oncology clinic, the surface samples for cyclophosphamide remained positive for 3 days, even after housekeeping staff cleaned the surfaces [NIOSH 2012]. It is possible that there was a spill that was either not reported or not cleaned appropriately. It is also possible that contamination may have spread by using the same mop and water bucket in the chemotherapy administration area to clean other areas of the specialty hospital. Additionally, it is possible that the contamination was tracked out of the chemotherapy administration area on a day we did not sample or from an area that we did not sample.

Currently, no occupational exposure limits (OELs) exist for chemotherapy drugs on surfaces or in the air. However, considering the carcinogenicity of these chemotherapy drugs, precautions to eliminate or minimize exposures are appropriate [IARC 2012]. 


\section{Employee Interviews and OSHA Injury/IIIness Logs}

Among the 15 staff we interviewed, 3 were board-certified veterinary oncologists, 4 were licensed veterinary technicians, and 8 were unlicensed veterinary aides who received on the job training by staff at this hospital or previous places of employment. A total of 12 were female and 3 were male. Average age was 30 years old, and average length of employment at the hospital was 3.6 years (median 2.5 years). Staff typically averaged working just over 40 hours a week.

We asked participants about a variety of medical conditions that could be associated with exposure to chemotherapy drugs, such as adverse reproductive outcomes, blood disorders, and kidney or liver disorders. No employees reported any of these conditions. We also asked about a variety of zoonotic diseases, such as leptospirosis, Campylobacter, parasitic infections, ringworm, and methicillin-resistant staphylococcus aureus (MRSA). MRSA may be a zoonotic infection but infection more commonly results from human to human transmission in the community. One employee reported a prior MRSA infection where the source of infection was not identified.

We asked employees who were currently working with chemotherapy drugs about a wide variety of nonspecific symptoms that may be related to working with chemotherapy drugs, such as headache, skin rashes, dizziness, facial flushing, unusual tiredness, and eye or respiratory irritation. No employees reported symptoms while working with or near chemotherapy drugs.

The hospital had established a comprehensive in-house training program for new employees whose duties involved working with or around chemotherapy drugs or chemotherapy patients. All hospital duties were included, from administrative to all aspects of patient care and diagnostic workups. This training had a skills assessment and approval by both the training coordinator and senior technician before employees performed duties on their own and progressed to the next level of training. Most employees reported being very satisfied with the hospital's present health and safety program, and they felt that training had improved over time, especially for new hires. Some employees expressed that they would like more training on potential human health effects of working with hazardous drugs. Most employees reported always wearing required PPE when handling chemotherapy drugs or working with patients with known or suspected zoonotic diseases. Employees reported that gloves rated for use with chemotherapy drugs were not available, gowns were changed daily, and N95 respirators were changed monthly.

We reviewed OSHA Logs from 2013-2016. We summarize this information in Tables 2 and 3. The incidents that resulted in days away from work or job transfer/restriction as reported on OSHA Logs:

2013: No days away from work or job transfer/restriction were recorded.

2014: One employee missed one day of work and had one day of job transfer or restriction due to getting aerosolized bleach in their eyes.

2015: One employee missed two days of work and had seven days of job transfer or restriction due to a slip/trip/fall. Another employee missed one day of work due to a slip/trip/fall. 
2016: One employee had seven days job transfer or restriction for a bite wound.

One employee had one day away from work and thirty days job transfer or restriction for a back strain. One employee had three days away from work and sixty days job transfer or restriction for a back strain.

Table 2. Summary of hospital OSHA Logs for years 2013-2016

\begin{tabular}{lccccc}
\hline Year & $\begin{array}{c}\text { Average } \\
\text { number of } \\
\text { employees }\end{array}$ & $\begin{array}{c}\text { Total number of } \\
\text { cases with days } \\
\text { away from work }\end{array}$ & $\begin{array}{c}\text { Total days } \\
\text { away from } \\
\text { work }\end{array}$ & $\begin{array}{c}\text { Total number of } \\
\text { cases with job } \\
\text { transfer/ restriction }\end{array}$ & $\begin{array}{c}\text { Total days } \\
\text { job transfer/ } \\
\text { restriction }\end{array}$ \\
\hline $2013^{*}$ & 60 & 0 & 0 & 0 & 0 \\
2014 & 74 & 1 & 2 & 1 & 1 \\
2015 & 91 & 2 & 3 & 1 & 7 \\
$2016 \dagger$ & 98 & 2 & 4 & 3 & 97 \\
\hline
\end{tabular}

*June-December 2013 reviewed due to corporate restructuring of the hospital

†January-November 2016 (date of our site visit)

Table 3. Types of injuries and illnesses reported on OSHA Logs 2013-2016

\begin{tabular}{lcccc}
\hline Type of injury/illness & 2013 & 2014 & 2015 & 2016 \\
\hline Patient bite/scratch & 4 & 23 & 34 & 23 \\
Slip/trip/fall & 0 & 0 & 6 & 1 \\
Strain/sprain & 0 & 1 & 1 & 3 \\
Needlestick/cut & 0 & 0 & 4 & 0 \\
Eye injury & 0 & 2 & 0 & 0 \\
Allergic reaction & 0 & 1 & 0 & 0 \\
Drug exposure & 0 & 0 & 2 & 1 \\
Bumped body part/injury & 0 & 0 & 0 & 4 \\
\hline
\end{tabular}


The hospital had comprehensive procedures in place for employees concerning accident prevention, response, and recordkeeping. Employees were required to report all injuries to staff or clients to their supervisor and to complete an incident report form. Patient bites and scratches were the most common incidents reported on the OSHA Logs for all years we reviewed. Hospital staff told us they recorded all patient bites and scratches on the OSHA Logs regardless of severity or whether medical attention was needed or not. OSHA requires reporting on OSHA Logs when the following conditions apply:

- Death

- Loss of consciousness

- Days away from work

- Restricted work activity or job transfer

- Medical treatment beyond first aid

Additional, specific conditions that require OSHA reporting and directions on completing OSHA Logs are located at https://www.osha.gov/recordkeeping/osha-rkforms-winstr fillable.pdf.

\section{Review of Hazardous Drug Training and Procedures}

Chemotherapy drug exposure in healthcare settings primarily happens through inhalation and dermal contact. Ingestion from contaminated surfaces and hand-to-mouth contact could happen, as well as unintentional injection through a needle-stick or sharps injury. Inhalation exposure may occur from airborne droplets, vapors, or dust. We observed the risk of inhalational exposure to be low. Dermal exposure may occur when employees touch contaminated surfaces, and oral exposure may occur from hand-to-mouth transmission. Dermal exposure for most employees in the specialty hospital appeared to be minimal because of their use of gloves and gowns.

The standard operating procedures for handling hazardous drugs provided adequate guidance to pharmacy and nursing personnel on safe techniques in preparing, administering, and disposing of hazardous drugs as well as managing chemotherapy drug spills.

The Class II, Type A BSC was serviced in June 2016 and was reported to be operating effectively by the servicing company. The average face velocity was 92 feet per minute (range: $80-100$ feet per minute). The BSC was scheduled for manufacturer's recommended maintenance and certification every six months.

\section{Conclusions}

We found no chemotherapy drug contamination in surface wipe samples collected throughout the chemotherapy administration area, the inside of a laundry bag, or outside of the chemotherapy transport bag. We detected cyclophosphamide on the floor just outside the chemotherapy administration area. The facility's standard operating procedures for handling hazardous drugs provide adequate guidance on safe techniques in preparing, administering, and disposing of hazardous drugs as well as managing chemotherapy drug spills. Although 
no OSHA or other recommended standards exist, considering the carcinogenicity of chemotherapy drugs, precautions to eliminate or minimize exposures are appropriate. Employees did not report health symptoms or past known infection with zoonotic diseases and were satisfied with the hospital's current training program, especially for new hires.

\section{Recommendations}

On the basis of our findings, we recommend the actions listed below. We encourage forming a labor-management health and safety committee or working group to discuss our recommendations and develop an action plan. Those involved in the work can best set priorities and assess the feasibility of our recommendations for the specific situation at the specialty hospital.

Our recommendations are based on an approach known as the hierarchy of controls (Appendix B). This approach groups actions by their likely effectiveness in reducing or removing hazards. In most cases, the preferred approach is to eliminate hazardous materials or processes and install engineering controls to reduce exposure or shield employees. Until such controls are in place, or if they are not effective or feasible, administrative measures and PPE may be needed.

\section{Administrative Controls}

The term administrative controls refers to employer-dictated work practices and policies to reduce or prevent hazardous exposures. Their effectiveness depends on employer commitment and employee acceptance. Regular monitoring and reinforcement are necessary to ensure that policies and procedures are followed consistently.

1. Require employees to use the BSC for all chemotherapy drug preparation. Never handle open vials of drug outside of the cabinet.

2. Continue training all new employees who may have contact with chemotherapy drugs about safe handling techniques and the potential health effects associated with exposure to chemotherapy drugs. Consider offering additional refresher training on potential health effects from hazardous drugs.

3. Provide training for housekeeping employees who enter the chemotherapy administration area. Place an emphasis on the hazards of drug residue, the importance of personal protective equipment, and the importance of following cleaning protocols. Instruct employees to never push the air out of trash bags before removing them from trash cans.

4. Use a cart to move chemotherapy drugs throughout the building. Even if the box is easily carried, a cart provides stability and makes navigation through doors easier while reducing the chances of dropping the package.

- Employees should remove gloves after the package is on the cart and when moving throughout the building to minimize the possibility of contaminating door handles.

- Employees should have chemotherapy protective gloves on the cart in case the 
package needs to be handled in transit.

- Employees should put on chemotherapy protective gloves when the cart and package are in the chemotherapy administration area prior to putting the chemotherapy drug away.

5. Ensure that the grill of the BSC is never blocked by chemotherapy transport bags. Instruct employees to keep the bag fully inside the cabinet.

6. Wash hands after removing the gloves used to handle chemotherapy drugs.

7. Use dedicated cleaning supplies, including mop buckets and mop heads, for the chemotherapy administration area. If possible, store this equipment in the same area where it is used.

8. Store the spill kits closer to the ground, and not on the top shelf, to prevent the need to reach up for the kits.

9. Identify patients that have received chemotherapy with brightly colored disposable collars or bands to alert staff that their vomit, urine, and feces may contain chemotherapy drugs and that these potentially contaminated areas should be cleaned according to standard operating procedures.

10. Dispose of glass slides used during chemotherapy administration in the chemotherapy sharps containers. Anything that has come into contact with a patient receiving chemotherapy treatment should be considered contaminated and disposed of appropriately.

11. Consider discontinuing footbath use outside isolation rooms and instead use disposable shoe covers. Place a trash can near the door of the isolation room to dispose of shoe covers when leaving the room.

12. Review and follow guidelines for reporting incidents on OSHA Logs. While all patient bites and scratches should receive first aid according to the hospital's written policies and be documented to determine rabies vaccination status of the patient, not all need reported on the OSHA Logs.

13. Contact your local OSHA consultation program for free additional assistance with PPE and other safety concerns. Identify your local OSHA office through their website at https://www.osha.gov/html/RAmap.html.

\section{Personal Protective Equipment}

PPE is the least effective means for controlling hazardous exposures. Proper use of PPE requires a comprehensive program and a high level of employee involvement and commitment. The right PPE must be chosen for each hazard. Supporting programs, such as training, change-out schedules, and medical assessment, may be needed. PPE should not be the sole method for controlling hazardous exposures. Rather, PPE should be used until effective engineering and administrative controls are in place.

1. Wear gloves rated for use with chemotherapy drugs. Inspect gloves for defects before use and change gloves on a regular basis. Gloves should conform to ASTM International D6978-05 (formerly American Society for Testing and Materials) 
Standard Practice for Assessment of Resistance of Medical Gloves to Permeation by Chemotherapy Drugs. Changing recommendations vary from 30-60 minutes [ASHP 2006; NIOSH 2016]. Whenever gloves are damaged or contact with a drug is known or suspected, carefully remove and dispose of them properly.

2. Discontinue wearing N95 respirators for everyday chemotherapy administration. The BSC and CSTD are appropriate protections.

3. Use full length, cleanable face shields for splash protection. This can replace the use of safety glasses. 


\section{Appendix A: Tables}

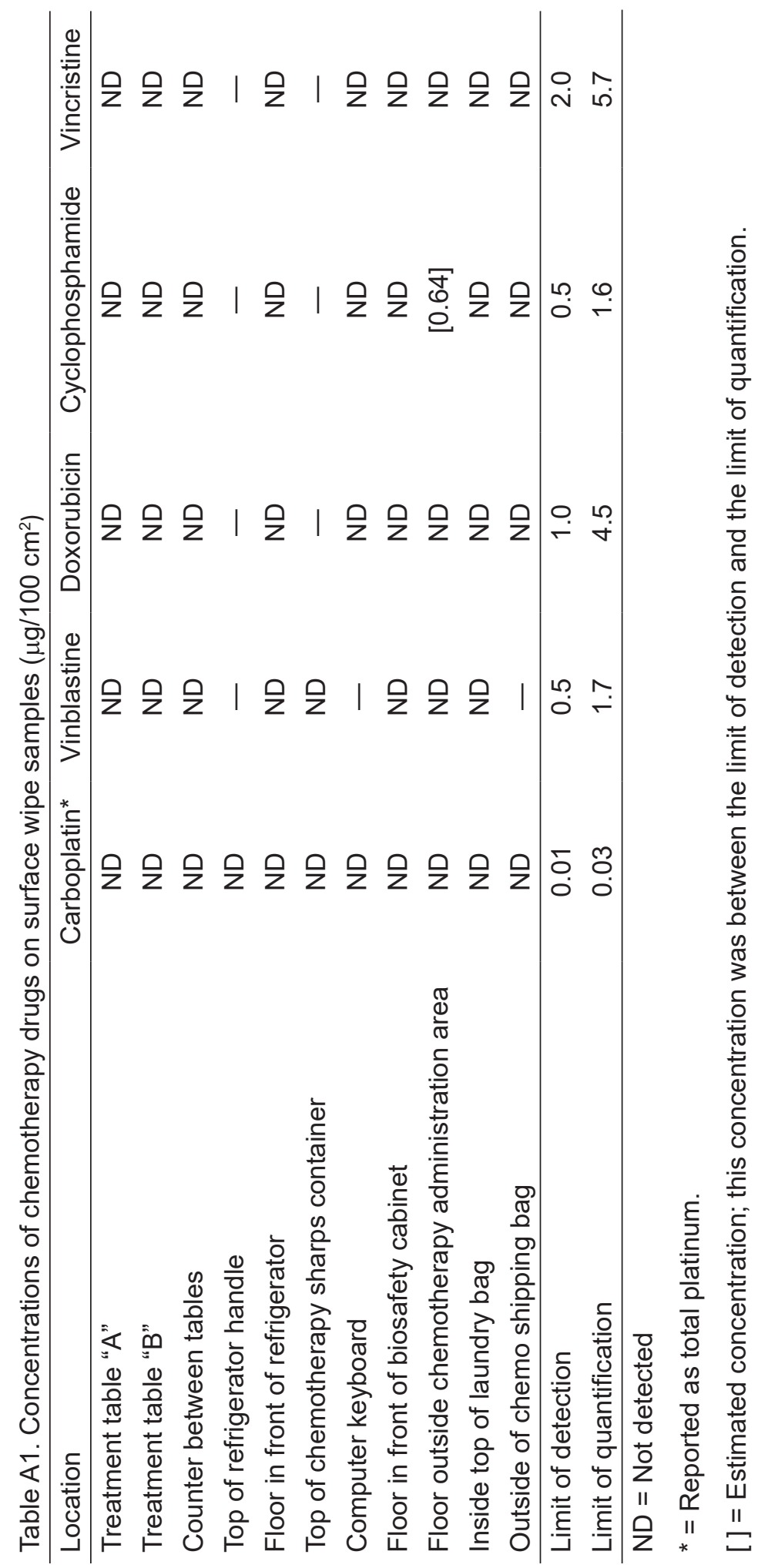




\section{Appendix B: Occupational Exposure Limits and Health Effects}

NIOSH investigators refer to mandatory (legally enforceable) and recommended OELs for chemical, physical, and biological agents when evaluating workplace hazards. OELs have been developed by federal agencies and safety and health organizations to prevent adverse health effects from workplace exposures. Generally, OELs suggest levels of exposure that most employees may be exposed to for up to 10 hours per day, 40 hours per week, for a working lifetime, without experiencing adverse health effects.

However, not all employees will be protected if their exposures are maintained below these levels. Some may have adverse health effects because of individual susceptibility, a preexisting medical condition, or a hypersensitivity (allergy). In addition, some hazardous substances act in combination with other exposures, with the general environment, or with medications or personal habits of the employee to produce adverse health effects. Most OELs address airborne exposures, but some substances can be absorbed directly through the skin and mucous membranes.

Most OELs are expressed as a time-weighted average (TWA) exposure. A TWA refers to the average exposure during a normal 8- to 10-hour workday. Some chemical substances and physical agents have recommended short-term exposure limits (STEL) or ceiling values. Unless otherwise noted, the STEL is a 15-minute TWA exposure. It should not be exceeded at any time during a workday. The ceiling limit should not be exceeded at any time.

In the United States, OELs have been established by federal agencies, professional organizations, state and local governments, and other entities. Some OELs are legally enforceable limits; others are recommendations.

- OSHA, an agency of the U.S. Department of Labor, publishes permissible exposure limits [29 CFR 1910 for general industry; 29 CFR 1926 for construction industry; and 29 CFR 1917 for maritime industry] called PELs. These legal limits are enforceable in workplaces covered under the Occupational Safety and Health Act of 1970.

- NIOSH recommended exposure limits (RELs) are recommendations based on a critical review of the scientific and technical information and the adequacy of methods to identify and control the hazard. NIOSH RELs are published in the NIOSH Pocket Guide to Chemical Hazards [NIOSH 2007]. NIOSH also recommends risk management practices (e.g., engineering controls, safe work practices, employee education/training, PPE, and exposure and medical monitoring) to minimize the risk of exposure and adverse health effects.

- Another set of OELs commonly used and cited in the United States include the threshold limit values or TLVs, which are recommended by the American Conference of Governmental Industrial Hygienists (ACGIH). The ACGIH TLVs are developed by committee members of this professional organization from a review of the published, peer-reviewed literature. TLVs are not consensus standards. They are considered voluntary exposure guidelines for use by industrial hygienists and others trained in this discipline "to assist in the control of health hazards" [ACGIH 2019]. 
Outside the United States, OELs have been established by various agencies and organizations and include legal and recommended limits. The Institut für Arbeitsschutz der Deutschen Gesetzlichen Unfallversicherung (Institute for Occupational Safety and Health of the German Social Accident Insurance) maintains a database of international OELs from European Union member states, Canada (Québec), Japan, Switzerland, and the United States. The database, available at https:/www.dguv.de/ifa/gestis/gestis-stoffdatenbank/index-2.jsp, contains international limits for more than 2,000 hazardous substances and is updated periodically.

OSHA (Public Law 91-596) requires an employer to furnish employees a place of employment free from recognized hazards that cause or are likely to cause death or serious physical harm. This is true in the absence of a specific OEL. It also is important to keep in mind that OELs may not reflect current health-based information.

When multiple OELs exist for a substance or agent, NIOSH investigators generally encourage employers to use the lowest OEL when making risk assessment and risk management decisions.

\section{Chemotherapy Drugs}

Currently the chemotherapy drugs we sampled have no OELs. Limited information exists that describes specific health effects due to occupational exposure of the drugs we tested. Some have been classified as carcinogenic by the International Agency for Research on Cancer (IARC). Doxorubicin is categorized as a Group 2A Carcinogen (inadequate evidence to designate it as a human carcinogen), and cyclophosphamide is categorized as a Group 1 Carcinogen (carcinogenic to humans) [IARC 2012]. Vincristine and vinblastine are categorized as Group 3 Carcinogens (not classifiable as to its carcinogenicity to humans). OSHA and NIOSH have not established OELs for platinum-containing chemotherapy drugs (like carboplatin) either as a group or as individual agents.

These drugs can be irritating to the eyes and respiratory tract, and some are considered a reproductive hazard and harmful to the fetus [Murff 2012; NIOSH 2004, 2016]. Workers who prepare and administer chemotherapy drugs may be at risk due to potential mutagenicity, teratogenicity, and/or carcinogenicity of these materials. Therefore, workplace exposures should be controlled to the lowest possible levels [NIOSH 2004, 2016]. 


\section{References}

ACGIH [2019]. 2019 TLVs $^{\circledR}$ and BEIs ${ }^{\circledR}$ : threshold limit values for chemical substances and physical agents and biological exposure indices. Cincinnati, OH: American Conference of Governmental Industrial Hygienists.

ASHP [2006]. American Society of Health-System Pharmacists guidelines on handling hazardous drugs. Am J Health Syst Pharm 63(12):1172-1191, https://doi.org/10.2146/ajhp050529.

Connor T, McDiarmid M [2006]. Preventing occupational exposures to antineoplastic drugs in health care settings. CA Cancer J Clin 56(6):354-365, https://doi.org/10.3322/canjclin.56.6.354.

Connor T, DeBord D, Pretty J, Oliver M, Roth T, Lees P, Krieg E Jr., Rogers B, Escalante C, Toennis C, Clark J, Johnson B, McDiarmid M [2010]. Evaluation of antineoplastic drug exposure of health care workers at three university-based US cancer centers. J Occup Environ Med 52(10):1019-1027, https://doi.org/10.1097/jom.0b013e3181f72b63.

CFR [2019]. Code of Federal Regulations. Washington, DC: U.S. Government Printing Office, Office of the Federal Register.

IARC [2012]. IARC monographs on the evaluation of the carcinogenic risk of chemicals to humans. Pharmaceuticals. Vol. 100A. Lyons, France: World Health Organization, International Agency for Research on Cancer, https://monographs.iarc.fr/wp-content/uploads/2018/06/mono100A.pdf.

Murff SJ [2012]. Safety and health handbook for cytotoxic drugs. Plymouth, United Kingdom: Government Institutes, ISBN 9781605907055.

NIOSH [2004]. NIOSH alert: preventing occupational exposures to antineoplastic and other hazardous drugs in health care settings. Cincinnati, OH: U.S. Department of Health and Human Services, Centers for Disease Control and Prevention, National Institute for Occupational Safety and Health, DHHS (NIOSH) Publication No. 2005-149, https://doi.org/10.26616/nioshpub2004165.

NIOSH [2007]. NIOSH pocket guide to chemical hazards. Cincinnati, OH: U.S. Department of Health and Human Services, Centers for Disease Control and Prevention, National Institute for Occupational Safety and Health, DHHS (NIOSH) Publication No. 2005-149, http://www.cdc.gov/niosh/npg/.

NIOSH [2012]. Chemotherapy drug exposures at an oncology clinic - Florida. By Couch J, West C. Cincinnati, OH: U.S. Department of Health and Human Services, Centers for Disease Control and Prevention, National Institute for Occupational Safety and Health, Health Hazard Evaluation Report 2009-0148-3158, https://www.cdc.gov/niosh/hhe/reports/pdfs/2009-0148-3158.pdf. 
NIOSH [2014]. Evaluation of chemotherapy drug exposure in an outpatient infusion center. By West C, Beaucham C. Cincinnati, OH: U.S. Department of Health and Human Services, Centers for Disease Control and Prevention, National Institute for Occupational Safety and Health, Health Hazard Evaluation Report 2013-0019-3205, https://www.cdc.gov/niosh/hhe/reports/pdfs/2013-0019-3205.pdf.

NIOSH [2016]. NIOSH list of antineoplastic and other hazardous drugs in healthcare settings, 2016. By Connor TH, MacKenzie BA, DeBord DG, Trout DB, O’Callaghan JP. Cincinnati, OH: U.S. Department of Health and Human Services, Centers for Disease Control and Prevention, National Institute for Occupational Safety and Health, DHHS (NIOSH) Publication No. 2016-161 (supersedes 2014-138), https://doi.org/10.26616/nioshpub2016161.

Stull J, Bjorvik E, Bub J, Dvorak G, Petersen C, Troyer H [2018]. 2018 AAHA infection control, prevention, and biosecurity guidelines. J Am Anim Hosp Assoc 54(6):1-30, https://www.aaha.org/public documents/guidelines/icpb_guidelines.pdf.

USP [2019]. <800> Hazardous drugs — handling in healthcare settings. Rockville, MD: U.S. Pharmacopeia.

https:/www.usp.org/compounding/general-chapter-hazardous-drugs-handling-healthcare. 
Keywords: North American Industry Classification System (NAICS) 541940 (Veterinary Services), South Carolina, Chemotherapy, Hazardous Drugs, Veterinary Clinic, Specialty Hospital, Animal Healthcare, Healthcare, Oncology 
This page left intentionally blank 
The Health Hazard Evaluation Program investigates possible health hazards in the workplace under the authority of the Occupational Safety and Health Act of 1970 (29 U.S.C. § 669(a) (6)). The Health Hazard Evaluation Program also provides, upon request, technical assistance to federal, state, and local agencies to investigate occupational health hazards and to prevent occupational disease or injury. Regulations guiding the Program can be found in Title 42, Code of Federal Regulations, Part 85; Requests for Health Hazard Evaluations (42 CFR Part 85).

\section{Disclaimer}

The recommendations in this report are made on the basis of the findings at the workplace evaluated and may not be applicable to other workplaces.

Mention of any company or product in this report does not constitute endorsement by NIOSH.

Citations to Web sites external to NIOSH do not constitute NIOSH endorsement of the sponsoring organizations or their programs or products. NIOSH is not responsible for the content of these Web sites. All Web addresses referenced in this document were accessible as of the publication date.

\section{Acknowledgments}

Analytical Support: Bureau Veritas North America, Inc.

Desktop Publisher: Shawna Watts

Editor: Cheryl Hamilton

Industrial Hygiene Field Assistance: Catherine Beaucham

Logistics: Donnie Booher, Kevin Moore

\section{Availability of Report}

Copies of this report have been sent to the employer and employees at the facility. The state and local health department and the Occupational Safety and Health Administration Regional Office have also received a copy. This report is not copyrighted and may be freely reproduced.

\section{Recommended citation for this report:}

NIOSH [2019]. Evaluation of chemotherapy drug exposure in a veterinary specialty hospital. By Grant MP, Gibbins J. Cincinnati, OH: U.S. Department of Health and Human Services, Centers for Disease Control and Prevention, National Institute for Occupational Safety and Health, Health Hazard Evaluation Report 2016-0231-3354, https://www.cdc.gov/niosh/hhe/reports/pdfs/2016-0231-3354.pdf. 
Delivering on the Nation's promise:

Promoting productive workplaces through safety and health research

To receive NIOSH documents or more information about occupational safety and health topics, please contact NIOSH:

Telephone: 1-800-CDC-INFO (1-800-232-4636)

TTY: 1-888-232-6348

CDC INFO: www.cdc.gov/info

or visit the NIOSH Web site at www.cdc.gov/niosh

For a monthly update on news at $\mathrm{NIOSH}$, subscribe to

$\mathrm{NIOSH}$ eNews by visiting www.cdc.gov/niosh/eNews. 